\title{
Improved Treatment Satisfaction with Dulaglutide and Insulin Glargine in Patients with T2D and Chronic Kidney Disease
}

\author{
Maria Yu, Fady T. Botros, Oralee J. Varnado, Kristina S. Boye
}

Eli Lilly and Company, Indianapolis, USA

\section{OBJECTIVE}

Patient reported outcomes were assessed to capture worry and behavioral changes associated with concerns and experiences of hypoglycemia as measured by the Adult Low Blood Sugar Survey (ALBSS). Patients' satisfaction with their diabetes treatment and perceived frequency of hyperglycemia and hypoglycemia was evaluated using the Diabetes Treatment Satisfaction Questionnaire (DTSQ).

Is there a difference in the hypoglycemia concerns or treatment satisfaction reported by patients with T2D and CKD treated with dulaglutide compared to glargine?

\section{STUDY DESIGN}

\begin{tabular}{|c|c|c|}
\hline \multirow{3}{*}{ 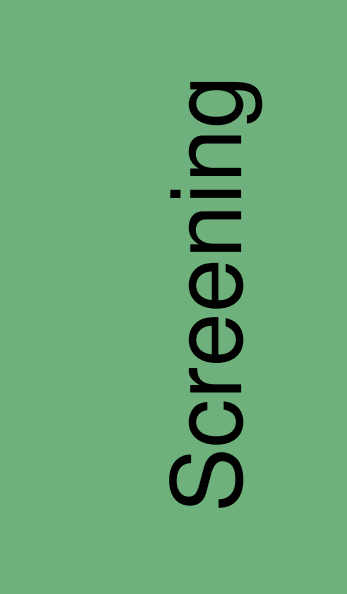 } & Dulaglutide $1.5 \mathrm{mg}$ once weekly + prandial lispro & \multirow{3}{*}{ 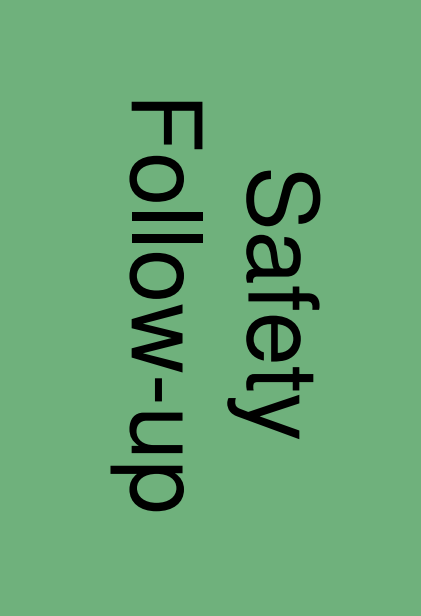 } \\
\hline & Dulaglutide $0.75 \mathrm{mg}$ once weekly + prandial lispro & \\
\hline & Insulin glargine + prandial lispro & \\
\hline Lead In & Treatment Period & Follow-up \\
\hline & 6 & \\
\hline
\end{tabular}

Randomization

Primary Endpoint

Final Endpoint

Insulin glargine dose was adjusted to target fasting plasma glucose (PG) values between $100-150 \mathrm{mg} / \mathrm{dL}$; insulin lispro dose were adjusted to target pre-prandial PG values between $120-180 \mathrm{mg} / \mathrm{dL}$. Additional study details previously reported.

\section{KEY RESULT}

\begin{tabular}{|c|c|c|c|c|}
\hline & Week & $\begin{array}{c}\text { Dulaglutide } 1.5 \mathrm{mg} \\
(\mathrm{N}=183)\end{array}$ & $\begin{array}{c}\text { Dulaglutide } 0.75 \mathrm{mg} \\
(\mathrm{N}=180)\end{array}$ & $\begin{array}{c}\text { Insulin glargine } \\
(\mathrm{N}=186)\end{array}$ \\
\hline \multicolumn{5}{|c|}{ Adult Low Blood Sugar Survey (ALBSS)a } \\
\hline \multirow{3}{*}{ Total Score } & Baseline & $23.3(16.78)$ & $22.0(18.00)$ & $25.4(17.92)$ \\
\hline & 26 & $-4.3(1.15)^{\star *}$ & $-3.1(1.13)^{\star}$ & $-1.6(1.09)$ \\
\hline & 52 & $-2.5(1.20)^{*}$ & $-3.8(1.19)^{\star}$ & $-3.5(1.17)^{*}$ \\
\hline \multirow{3}{*}{ Behavior Scale } & Baseline & $27.2(18.36)$ & $26.5(21.87)$ & $30.4(20.63)$ \\
\hline & 26 & $-4.3(1.40)^{*}$ & $-3.3(1.38)^{\star}$ & $-2.5(1.33)$ \\
\hline & 52 & $-2.1(1.44)$ & $-4.0(1.42)^{*}$ & $-3.5(1.40)^{*}$ \\
\hline \multirow{3}{*}{ Worry Scale } & Baseline & $20.1(20.92)$ & $18.5(19.21)$ & $21.4(21.23)$ \\
\hline & 26 & $-4.3(1.33)^{\star}$ & $-3.4(1.31)^{*}$ & $-1.1(1.26)$ \\
\hline & 52 & $-2.9(1.38)^{*}$ & $-4.2(1.37)^{*}$ & $-3.7(1.35)^{*}$ \\
\hline \multicolumn{5}{|c|}{ Diabetes Treatment Satisfaction Questionnaire (DTSQ) } \\
\hline \multirow{3}{*}{ Total Score } & Baseline & $27.5(7.32)$ & $28.3(7.02)$ & $26.7(8.19)$ \\
\hline & 26 & $4.5(0.46)^{\star *}$ & $4.0(0.46)^{\star *}$ & $4.0(0.44)^{\star *}$ \\
\hline & 52 & $4.1(0.48)^{\star *}$ & $3.6(0.48)^{\star \star}$ & $4.8(0.47)^{\star *}$ \\
\hline \multirow{3}{*}{ Hyperglycemia Scale } & Baseline & $3.5(1.84)$ & $3.5(1.81)$ & $3.4(1.88)$ \\
\hline & 26 & $-1.1(0.15)^{\star *}$ & $-1.0(0.15)^{\star \star \#}$ & $-1.4(0.15)^{\star \star}$ \\
\hline & 52 & $-1.1(0.16)^{\star *}$ & $-1.2(0.16)^{* *}$ & $-1.3(0.15)^{* *}$ \\
\hline \multirow{3}{*}{ Hypoglycemia Scale } & Baseline & $1.8(1.67)$ & $1.5(1.77)$ & $1.6(1.72)$ \\
\hline & 26 & $-0.1(0.13)$ & $-0.0(0.13)$ & $-0.1(0.13)$ \\
\hline & 52 & $-0.2(0.14)$ & $-0.2(0.14)$ & $0.0(0.13)$ \\
\hline \multicolumn{5}{|c|}{$\begin{array}{l}\text { ormed scores }(0 \text { to } 100) \text {. All baseline scores presented as mean (SD). All change from baseline scores presented } \\
\text { squares mean (SE). }{ }^{*},{ }^{* *} 2 \text {-sided } p<.05 \text { and } p<.001 \text { versus baseline; } \# 2 \text {-sided } p<.05 \text { vs insulin glargine. }\end{array}$} \\
\hline \multicolumn{5}{|c|}{$\begin{array}{l}\text { Ignificant decreases from baseline (improvements) in all } 3 \text { ALBSS scores were } \\
\text { oserved with dulaglutide at both } 26 \text { and } 52 \text { weeks (except for the Behavior } \\
\text { core at } 52 \text { weeks in the dulaglutide } 1.5 \mathrm{mg} \text { arm) and with glargine only at } 52 \\
\text { eeks }\end{array}$} \\
\hline \multicolumn{5}{|c|}{$\begin{array}{l}\text { gnificant increases from baseline (improvements) in the DTSQ Total score } \\
\text { d significant decreases from baseline (improvements) in the DTSQ } \\
\text { perglycemia score were observed with dulaglutide and glargine at } 26 \text { and } 52 \\
\text { eeks }\end{array}$} \\
\hline \multicolumn{5}{|c|}{$\begin{array}{l}\text { At } 26 \text { weeks, the decrease from baseline in the DTSQ Hyperglycemia score in the } \\
\text { dulaglutide } 0.75 \mathrm{mg} \text { arm was significantly smaller than the decrease in the insulin glargine } \\
\text { arm }\end{array}$} \\
\hline \multicolumn{5}{|c|}{$\begin{array}{l}\text { ulaglutide treatment and the implemented insulin dose adjustment algorithms } \\
\text { argeting fasting PG of } 100-150 \mathrm{mg} / \mathrm{dL} \text { and preprandial PG between } 120-180 \\
\mathrm{~g} / \mathrm{dL} \text { ) may have contributed to these improvements }\end{array}$} \\
\hline \multirow{2}{*}{\multicolumn{5}{|c|}{$\begin{array}{l}\text { Jo significant differe } \\
\text { DNCLUSION }\end{array}$}} \\
\hline & & & & \\
\hline \multicolumn{5}{|c|}{$\begin{array}{l}\text { latients with T2D and CKD si } \\
\text { ociated with less worry and } \\
\text { rovements in treatment sati } \\
\text { juency of hyperglycemia wit } \\
\text { aglutide and glargine. }\end{array}$} \\
\hline
\end{tabular}

\section{BACKGROUND}

- Many diabetes medications, including insulin, are primarily cleared by the kidneys; therefore, medications used to treat T2D often require dose adjustments or are contraindicated in patients with $\mathrm{CKD}^{2,3}$

- No dose adjustment for dulaglutide is needed for patients with varying degrees of renal impairment

- Beneficial clinical outcomes associated with dulaglutide and prandial insulin lispro combination therapy in patients with T2D and CKD can be further assessed through patient-reported outcome (PRO) measures

- ALBSS includes behavior and worry items; behavior items ask patients to rate how often they engaged in each behavior during their daily routine and worry items ask how often they worried about certain factors/events associated with low blood sugar ${ }^{4,5}$

-DTSQ evaluates absolute treatment satisfaction and includes scales to measure patients' perceived hypoglycemia and hyperglycemia ${ }^{6,7}$

\section{BASELINE CHARACTERISTICS}

\begin{tabular}{|c|c|c|c|}
\hline $\begin{array}{l}\text { Demographics (safety population, } \\
\text { except FBG [mIJTT population]) }\end{array}$ & $\begin{array}{l}\text { Dulag|utide } 1.5 \mathrm{mg} \\
\mathrm{N}=192\end{array}$ & $\begin{array}{l}\text { Dulaglutide } 0.75 \mathrm{mg} \\
\qquad \mathrm{N}=190\end{array}$ & $\begin{array}{c}\text { Insulin Glargine } \\
\mathrm{N}=194\end{array}$ \\
\hline Sex, women & $88(45.8)$ & $86(45.3)$ & $101(52.1)$ \\
\hline Age, years & $64.7 \pm 8.8$ & $64.7 \pm 8.6$ & $64.3 \pm 8.4$ \\
\hline Duration of diabetes, years & $17.6 \pm 8.7$ & $18.0 \pm 8.8$ & $18.7 \pm 8.7$ \\
\hline A1c, \% (mmol/mol) & $\begin{array}{c}8.6 \pm 0.9 \\
(70.5 \pm 9.8)\end{array}$ & $\begin{array}{c}8.6 \pm 1.1 \\
(70.5 \pm 12.0)\end{array}$ & $\begin{array}{c}8.6 \pm 1.0 \\
(70.5 \pm 10.9)\end{array}$ \\
\hline $\mathrm{FBG}, \mathrm{mg} / \mathrm{dL}(\mathrm{mmol} / \mathrm{L})$ & $\begin{array}{c}161.5 \pm 55.6 \\
(9.0 \pm 3.1)\end{array}$ & $\begin{array}{c}166.6 \pm 70.6 \\
(9.3 \pm 3.9)\end{array}$ & $\begin{array}{c}170.7 \pm 72.2 \\
(9.5 \pm 4.0)\end{array}$ \\
\hline Weight, kg & $88.1 \pm 16.0$ & $90.9 \pm 18.3$ & $88.2 \pm 18.5$ \\
\hline BMI, $\mathrm{kg} / \mathrm{m}^{2}$ & $32.1 \pm 4.8$ & $33.0 \pm 5.5$ & $32.4 \pm 5.3$ \\
\hline Daily total insulin dose, $U$ & $58.8 \pm 30.1$ & $56.6 \pm 31.2$ & $59.3 \pm 34.2$ \\
\hline Daily total insulin dose, $\mathrm{U} / \mathrm{kg}$ & $0.7 \pm 0.3$ & $0.6 \pm 0.3$ & $0.7 \pm 0.3$ \\
\hline Duration of CKD Stage 3 or higher, years & $4.2 \pm 5.6$ & $4.0 \pm 4.9$ & $3.5 \pm 4.0$ \\
\hline $\mathrm{eGFR}^{8}, \mathrm{ml} / \mathrm{min} / 1.73 \mathrm{~m}^{2}$ & $38.1 \pm 13.2$ & $38.3 \pm 12.3$ & $38.5 \pm 13.0$ \\
\hline $60 \leq$ Baseline eGFR $<90$ & $9(4.7)$ & $7(3.7)$ & $14(7.2)$ \\
\hline $45 \leq$ Baseline eGFR $<60$ & $53(27.6)$ & $53(27.9)$ & $51(26.3)$ \\
\hline $30 \leq$ Baseline eGFR $<45$ & $73(38.0)$ & $75(39.5)$ & $67(34.5)$ \\
\hline $15 \leq$ Baseline eGFR $<30$ & $55(28.6)$ & $55(28.9)$ & $61(31.4)$ \\
\hline Baseline eGFR $<15$ & $2(1.0)$ & $0(0.0)$ & $1(0.5)$ \\
\hline UACR, g/kg (mean [median]) & $779.1(213.7)$ & $842.2(233.6)$ & $919.5(195.6)$ \\
\hline Microalbuminuria $(30 \leq$ UACR $\leq 300)$ & $74(38.5)$ & $61(32.3)$ & $56(28.9)$ \\
\hline Macroalbuminuria $($ UACR $>300$ ) & $84(43.8)$ & $84(44.4)$ & $90(46.4)$ \\
\hline
\end{tabular}

Data presented as mean \pm SD or $n(\%)$, unless otherwise noted, MMRM analysis. eGFR was calculated based on the CKD-EP equation. Abbreviations: $A 1 \mathrm{c}=$ glycated $\mathrm{A} 1 \mathrm{c} ; \mathrm{BMI}=$ body mass index; $\mathrm{DU}=$ dulaglutide; $\mathrm{FBG}=$ fasting blood glucose.

\section{ADDITIONAL RESULTS}

Glycemic measures at 26 and 52 weeks

\begin{tabular}{|c|c|c|c|c|}
\hline & Weeks & $\begin{array}{l}\text { Dulaglutide } 1.5 \mathrm{mg} \\
\text { (N=183) }\end{array}$ & $\begin{array}{c}\text { Dulaglutide } 0.75 \mathrm{mg} \\
(\mathrm{N}=180)\end{array}$ & $\begin{array}{l}\text { Insulin glargine } \\
(\mathrm{N}=186)\end{array}$ \\
\hline \multirow{2}{*}{$\triangle$ HbA1c, $\%$} & 26 & $-1.2(0.1)^{\dagger+* *}$ & $-1.1(0.1)^{\dagger \star \star}$ & $-1.1(0.1)^{\star *}$ \\
\hline & 52 & $-1.1(0.1)^{\dagger \dagger * *}$ & $-1.1(0.1)^{\dagger \star *}$ & $-1.0(0.1)^{\star \star}$ \\
\hline \multirow{2}{*}{$\Delta \mathrm{HbA} 1 \mathrm{c}, \mathrm{mmol} / \mathrm{mol}$} & 26 & $-13.1(1.1)^{\dagger \star \star \star}$ & $-12.0(1.1)^{\dagger \star \star}$ & $-12.0(1.1)^{\star \star}$ \\
\hline & 52 & $-12.0(1.1)^{\dagger \dagger \star *}$ & $-12.0(1.1)^{\dagger \star \star}$ & $-10.9(1.1)^{\star \star}$ \\
\hline \multirow{2}{*}{$\Delta$ Weight, kg } & 26 & $-2.8(0.4)^{\star \star \# \#}$ & $-2.0(0.4)^{* \star \# \#}$ & $1.1(0.3)^{*}$ \\
\hline & 52 & $-2.7(0.5)^{\star \star \# \#}$ & $-1.7(0.4)^{\star \star \# \#}$ & $1.6(0.4)^{\star \star}$ \\
\hline \multirow{2}{*}{$\begin{array}{l}\text { Total Hypoglycemia rate, } \\
\text { events/participant/yeara }\end{array}$} & 26 & $5.5 \pm 12.5^{\# \#}$ & $7.8 \pm 20.4^{\# \#}$ & $17.1 \pm 27.7$ \\
\hline & 52 & $5.8 \pm 13.7^{\# \#}$ & $7.6 \pm 17.8^{\#}$ & $14.4 \pm 22.2$ \\
\hline \multirow{2}{*}{$\begin{array}{l}\text { Documented Symptomtic } \\
\text { Hypoglycemia rate, } \\
\text { events/participant/year }\end{array}$} & 26 & $4.2 \pm 11.6^{\# \#}$ & $4.9 \pm 13.4^{\# \#}$ & $11.3 \pm 22.0$ \\
\hline & 52 & $4.4 \pm 12.2^{\#}$ & $4.3 \pm 9.3^{\# \#}$ & $9.6 \pm 17.7$ \\
\hline
\end{tabular}

aplasma glucose $\leq 70 \mathrm{mg} / \mathrm{dL}$. Values presented as least squares mean (SE) for $\mathrm{HbA1c}$ and weight or mean $\pm \mathrm{SD}$ for

hypoglycemia.
$t,+t$ Multiplicity adjusted 1 -sided $p<0.001$ for noninferiority versus insulin glargine with a $0.4 \%$ margin or $0.3 \%$ margin, respectively; * ** 2 -sided $p<0.05$ or $p<.001$ vs baseline, respectively; \#, \# 2 -sided $p<0.05$ or $p<.001$ vs insulin glargin

References:

1. Tuttle et al. Poster presented at ADA 2017. Abstract LB36 (138-LB)

2. National Kidney Foundation. Am J Kidney Dis. 2012;60(5):850-886.

3. Tuttle et al. Am J Kidney Dis. 2014;64(4):510-533.

4. Cox et al. Diabetes Care. 1987;10(5):617-21

5. Stargardt et al. HQOL. 2009;7:91. doi:10.1186/1477-7525-7-91.

6. Bradley C. In: Handbook of Psychology and Diabetes. 1994; 111-32.

7.Bradley C. Diabetes Care. 1999;22(3):530-2

8. Levey et al. Ann Intern Med. 2009;150(9):604-612
Scan for poster and other disclosures
presented at IDF 2017 政 